\title{
Factores de Liderazgo en la Fiebre del Oro en la Región Sur del Ecuador
}

\author{
Manuel U. Ordoñez ${ }^{1}$, Miguel A. Bustamante ${ }^{2^{\star}}$ y Roberto M. Campos ${ }^{2,3}$ \\ (1) Universidad Católica de Santiago de Guayaquil. Programa de Maestría en Administración de \\ Empresas. Av. Carlos Julio Arosemena Km. 11/2 vía Daule, Guayaquil, Ecuador. (e-mail: \\ jklmanuel@gmail.com) \\ (2) Escuela de Ingeniería Comercial, Facultad de Economía y Negocios, Universidad de Talca, \\ Campus Lircay, Avenida Lircay S/N, Talca-Chile (e-mail: mabu@utalca.cl) \\ (3) Escuela de Negocios EGEU, Programa de Doctorando, Eliodoro Yañez 869, Santiago-Chile (e-mail: \\ rcampos@utalca.cl)
}

${ }^{*}$ Autor a quien debe ser dirigida la correspondencia

Recibido Oct. 28, 2016; Aceptado Ene. 6, 2017; Versión final Mar. 3, 2017, Publicado Jun. 2017

\section{Resumen}

Se determinan y analizan los rasgos de personalidad de los líderes, las percepciones de los seguidores y los aspectos de percepción de la cultura organizacional en el sector de exploración y explotación de recursos no renovables como el oro y otros minerales, en una compañía de la Provincia de El Oro del Ecuador. Se aplicó un cuestionario con 45 preguntas en escala Likert. Se realizaron las medidas de confiabilidad, KMO y coeficientes de significación, además de un análisis de factores de componentes principales con rotación varimax. Se concluye que los colaboradores tienen confianza en el liderazgo, existe comunicación con sus jefaturas y sienten el apoyo de sus líderes. Además, valoran la participación que se les otorga, el buen ambiente de trabajo y la relación igualitaria de sus jefes. Aprecian que la personalidad de sus líderes se explica por tener que enfrentar actividades de alto riesgo.

\section{Factors of Leadership in the Gold Rush in the South Region of the Ecuador}

\begin{abstract}
The features of the leaders' personality, the perceptions of the followers and the aspects of perception of the organizational culture in the exploration sector and exploitation of non-renewable resources as the gold and other minerals, in a company of the County of Gold of the Ecuador are determined and analyzed. A questionnaire was applied with 45 questions in the Likert scale. Also, were carried out the measures of dependability, KMO and significance coefficients, besides an analysis of factors of main components with varimax rotation. It is concluded that the collaborators trust the leadership, communication exists with their headquarters and they feel the support of their leaders. Also, they value the participation that they are granted, the good working atmosphere and the equitable relationship of their bosses. They appreciate that the personality of their leaders is explained by the high risk activities they perform.
\end{abstract}

Keywords: features of the leadership; perceptions of leadership; organizational culture; transformational leadership 


\section{INTRODUCCIÓN}

En general, el liderazgo se refiere a la influencia que ejerce una persona sobre un grupo de referencia e impacta la eficacia del colectivo (Pedroja-Rejas y Rodriguez-Ponce, 2008). Así mismo, el hecho de brindar servicios a cambio de la satisfacción interpersonal es bien conocido como liderazgo transaccional, en tanto que, el liderazgo transformacional (Avolio y Bass, 2004), es reconocido como uno de los estilos en el cual confluyen la estimulación intelectual, cambios en la forma de pensar, inspiración, carisma, confianza, respeto y carácter excepcional del líder para la conducción estratégica de las organizaciones (Bass y Avolio, 2003). Un factor importante del liderazgo en la actual economía globalizada, es cómo contribuye a la innovación y la manera en que las empresas se gestionan para lograr dicha innovación. El liderazgo ayuda al diseño de políticas de incentivos adecuadas y eficientes (Cabaleiro, et al., 2016) y contribuye a generar un desarrollo de competencias positivas, dentro del liderazgo como el observado en algunas empresas colombianas (Ochoa y Ríos, 2011) que las han transformado en gigantes empresariales.

Diversos estudios de liderazgo se han abierto al análisis de los denominados factores de percepción de los colaboradores respecto a sus líderes (Hollander, 1978, citado por Terroni, 2002; Bass, et al., 2003) en tanto que paralelamente se han estudiado las vivencias e interrelaciones que las personas sostienen con sus líderes (Avolio y Bass, 2004) y que van desde aquellos que demuestran conductas de tipo democrático hasta quienes ejercen un estilo autoritario (Robbins, 1996). No obstante, el liderazgo y la dirección pueden encontrarse en un punto de unión complementario, que hace a los directivos más eficaces en la gestión de las organizaciones (Pautt, 2011). En consecuencia, el líder a partir de sus conocimientos y experiencia determina un modelo de liderazgo propio, el cual usa para dirigir a las personas con el objetivo de conformar equipos de alto desempeño para alcanzar metas de forma eficiente, mediante la coordinación y estructuración adecuada de sus equipos (Escalona-Barbosa y Hurtado-Ayala, 2016).

Por otra parte, el ambiente y la cultura producen un entorno dinámico y complejo que trasciende y que transforma relaciones y conductas, entre otros, del propio líder (Robbins, 1996) dando pie a un liderazgo que no sólo refleja conductas inherentes a la naturaleza humana, transformando al líder y a los seguidores (Morelos-Gomez y Fontalvó-Herrera, 2014) sino que también se ve influenciado por un sinnúmero de variables derivadas del contexto ambiental, político y socioeconómico que pueden impactar positiva o negativamente en el medio en cual se insertan las organizaciones (Peterson y Luthans, 2003; Piccolo y Colquitt, 2006). Es por ello que el líder debe ser visto como un agente del cambio con aptitudes, actitudes, conocimientos, habilidades personales y capacidad de dirección, entre otros rasgos, que le permitan cambiar la dinámica organizacional en la que ejercen los subordinados (Gil e lbarra, 2014).

Además, se afirma que el rendimiento efectivo del grupo depende de la armonía que se produzca entre el estilo del líder para interactuar con sus subordinados y el grado de control que se tenga de la influencia que la situación y contexto afecten al líder (Morelos-Gomez y Fontalvó-Herrera, 2014), dando cabida a la teoría de los recursos cognoscitivos que actualiza el modelo de contingencia de Fiedler citado por Robbins (1996). Es preciso entonces, comprender el liderazgo como una actitud de compromiso de la dirección hacia sus trabajadores y el entorno social, el cual debe ser adecuado para que los trabajadores utilicen al máximo sus habilidades y conocimientos (Perez, 2012) haciendo que los equipos de trabajo y sus líderes funcionen como una totalidad no fragmentada (Fardella, 2013) capaz de generar rendimientos organizacionales superiores. Otros estudios por su parte, focalizan la relación entre el rendimiento organizacional y las actitudes del líder (Robbins, 1996), avanzando progresivamente hacia el análisis de contextos, situaciones y relaciones de acuerdo con las teorías de Liao y Chuang (2004) y de Li y Liao (2014), generándose la apreciación y valoración de dos concepciones de liderazgo, el transformacional y el transaccional, por cuanto han recibido la mayor atención en los últimos años y se ubican entre las teorías emergentes (Garcia, 2015)

El liderazgo transaccional opera con base en la autoridad burocrática y legitimidad del puesto dentro de la organización (Godoy y Bresó, 2013) y gestiona el cumplimiento de sus propósitos a través del manejo por excepción activa de estándares de control del desempeño aplicando correcciones a las desviaciones encontradas (Salinas y Cordero, 2016), es decir, el control implica intervenciones del líder cuando se presentan dificultades de cumplimiento de objetivos (Perez y Camps, 2011). El liderazgo transformacional sin embargo, motiva a los colaboradores a través de la inspiración, alentando su desarrollo individual para asumir retos y alcanzar los propósitos colectivos. Este estilo, se considera más efectivo y está positivamente relacionado con el desempeño, la satisfacción laboral y la motivación hacia el trabajo de los subordinados (Espinosa, et al., 2015).

Es así como con el tiempo, diversos estudios han generado una cierta taxonomía que define a lo menos dos supuestos claves, a) que los líderes inteligentes y competentes formulan una mayor cantidad de planes, decisiones y estrategias de acción más efectivos que los líderes menos inteligentes, y b) que los gerentes o 
jefes comunican sus planes, decisiones y estrategias por medio de conductas directivas (Morelos-Gomez y Fontalvó-Herrera, 2014), que determinan el funcionamiento más eficaz posible de las organizaciones en sus respectivos contextos (Lim y Ployhart, 2004; Luthans, et al., 2005).

Así por ejemplo, analizadas las representaciones sociales del liderazgo en gerentes venezolanos, se observa que los gerentes se caracterizan por la figura del líder basado en sus particularidades afirmándose que el liderazgo es una competencia (Borjas, 2011). Sin embargo, hoy las condiciones de dinamismo y globalización de los mercados, especialmente el mercado laboral minero y su organización a nivel internacional implican nuevos desafíos en modelos de liderazgo. En este ámbito el Liderazgo transaccional es un factor relevante por cuanto establece relaciones de intercambio de bienes y servicios entre el líder y sus subordinados (Salinas y Cordero, 2016).

Desde una perspectiva más bien sectorial, un estudio destinado a identificar predictores de excelencia desde la voz del empleado (EVE) en un centro hospitalario, determinó que tres de los factores resultaron significativos en la regresión, entre éstos el liderazgo directivo con una capacidad de explicación de 34.8\%, afirmándose que éste modelo ofrece posibilidades de uso en otros contextos (Perez-Rave, et al., 2013) como podría ser el de la minería. Por otra parte, y volviendo a los estudios clásicos, el denominado Grid Gerencial de Blake y Mounton citados por Robbins (1996), sintetiza una red de estilos de liderazgo que entrecruza el interés del líder por las personas con la preocupación del mismo por la producción, produciendo 81 combinaciones de liderazgo; sin embargo para las organizaciones mineras, la innovación es un factor importante en tanto debe hacer frente a una dinámica del entorno que se expresa en nuevos escenarios sociales, económicos, comerciales y tecnológicos, que modifican las configuraciones estructurales y los procesos de dirección, por supuesto utilizando la innovación permanente para que las empresas se mantengan competitivas (Turbay-Posada, 2013)

Surge así un liderazgo estratégico clave y esencial a las organizaciones, que releva los factores de personalidad de los líderes como referentes de estilos perfectamente identificables para la activación de las capacidades de los subordinados, sea éste ejercido en sus versiones transaccional o transformacional (Wegner, 2004), teniendo presente que con ello se actúa sobre los denominados factores críticos del éxito que provienen del liderazgo como fuerza impulsora y reguladora del poder de adaptación de los talentos organizacionales, a través de los cuales la empresa centra su atención en lo estratégicamente importante. (Gil e Ibarra, 2014)

De lo indicado y, siguiendo los postulados también tradicionales de la teoría del liderazgo situacional de Hersey y Blanchard de 1951 citado por Robbins (1996), el foco de la gestión gira en torno a los seguidores y en cómo el líder escoge un estilo adecuado de liderazgo que sea coherente con el grado de madurez de los seguidores que se manifiesta en la capacidad y voluntad de las personas para asumir responsabilidades y guiar su propia conducta (Wegner, 2004; Seibert, et al., 2011). En general, los modelos de liderazgo muestran una transición progresiva que avanza entre diversos paradigmas que buscan explicar las relaciones líder - subordinados así como también comprender, desde la dinámica del cambio, cómo se ha transitado desde modelos estáticos que se definen sobre la base de la teoría de los rasgos, hacia aquellos modelos dinámicos, como son los de transformación y conducción estratégica de las organizaciones (Robbins, 1996).

Desde una perspectiva más contemporánea, se ha incorporado el hecho que los tiempos actuales requieren de nuevos estilos de liderazgo, más flexibles, abiertos y dinámicos y, así mismo, de nuevos líderes, tal vez con nuevos rasgos (Wang, et al., 2013). Es así como desde la década del 90, se cuenta con estudios que contrastan, mediante análisis de factores de componentes principales, comportamientos definidos como clave. Se identifican de esta forma los estilos de liderazgo transformacional con 3 factores que lo definen: el carismático-inspiracional, el intelectualmente estimulante y el que propicia la consideración individual (Gil e Ibarra, 2014). Complementariamente y, a modo de contraste teórico, los estudios de las dimensiones del liderazgo transaccional, han identificado también 3 factores o modos de expresión de este estilo, a decir: los de recompensa contingente, dirección por excepción y laissez-faire, dando espacio a una comprensión tricotómica de estos estilos (Wegner, 2004).

Análisis posteriores corroboran los hallazgos obtenidos en los estilos de liderazgo mencionados concluyendo en la existencia de un liderazgo activo, un segundo pasivo y un tercer factor de empowerment, separado del factor de liderazgo laissez-faire (Dvir, et al., 2002; Whetstone, 2002). En síntesis, si bien el líder transformacional requiere de holguras, porque es preciso invertir tiempo y energía para crear confianza y convencer a los seguidores de creer en una visión compartida, existen también líderes que ejercen la gestión desde una perspectiva egocéntrica, de explotación; sea porque están más bien orientados al poder o porque sus propios principios morales son más bien utilitarios (Ehrhart, 2004; Avolio y Bass, 2004). 
En general, los líderes transformacionales necesitan para el ejercicio de su liderazgo asumir la motivación de sus seguidores (Gil e lbarra, 2014), pero no siempre incorporan la dinámica situacional y los efectos contextuales y, por lo general, asumen que los seguidores quieren trabajar junto a ellos dirigidos hacia un objetivo de mayor envergadura (Avolio y Bass, 2004). Por una parte, porque el liderazgo tiene finalmente un efecto decisivo y considerable sobre la cultura de las organizaciones, y en consecuencia, si el líder es percibido distante de sus subordinados, puede tener un impacto negativo sobre la cultura y la confianza (Morelos-Gomez y Fontalvó-Herrera, 2014) lo que ha sido demostrado a nivel internacional por cuanto existe una relación entre estilos de liderazgo y cultura organizativa. El estilo de liderazgo explica el 46,2\% de la cultura de innovación y un $73,1 \%$ de la cultura competitiva. Asimismo, existe una relación entre cultura organizativa y eficacia, explicando esta última en un 69,9\% por la cultura (Rodríguez, 2010).

Por otra parte, el cambio y la dinámica de los escenario organizacionales y competitivos, hace necesario que los líderes enfrenten situaciones donde los seguidores no siempre disponen de las habilidades, destrezas o la experiencias necesarias para completar una tarea o, simplemente, no están motivados para llevar a cabo actividades sin una recompensa inmediata y tangible (Avolio y Bass, 2004) y donde la influencia del líder puede no ser el esperado, sino más bien expresarse de manera modesta dependiendo de las relaciones y patrones de comportamiento entre líder y subordinados (Theime y Treviño, 2012), lo cual se traduce en impactos psicológicos que han sido también estudiados en equipos organizacionales debidamente empoderados (Seibert, et al., 2011) generando orientaciones que contribuyen a definir relaciones productivas y dinámicas como las que propicia y resuelve el liderazgo transformacional (Wegner, 2004; Walumbwa, et al., 2004).

Ahora bien, asumiendo algunas de las perspectivas más innovadoras de estudio del liderazgo, desde la investigación documental con enfoque hermenéutico, se describen algunas teorizaciones del liderazgo modernista y postmodernista, afirmándose que el liderazgo tiene su centro en el ser humano, en consecuencia, ante el quiebre del modo de pensar modernista, no resuelto por los argumentos postmodernistas, se requiere de un retorno a los referentes básicos, es decir, al hombre y sus principios y con ello, a un liderazgo centrado en la persona humana integral dotada de cuerpo y espíritu (Mendoza, 2008).

Así por ejemplo, los directivos colombianos muestran, en general, avances de liderazgo relevantes (Duran y Castañeda, 2015) y, en particular, en Bucaramanga (Contreras, et al., 2016) al tiempo que, de algunos estudios comparativos entre Colombia y México, se observan patrones similares de liderazgo pero con algunas diferenciaciones menores. Para los colombianos, la práctica de liderazgo transformacional menos frecuente fue la de inspirar, mientras que la más frecuente fue la de modelar. Sin embargo, se observaron diferencias significativas en modelar el camino y dar aliento al corazón, estructurándose prácticas del liderazgo transformacional y transaccional respectivamente. Además se confirma el hecho que los directivos mexicanos, aunque tienen sus propias características, en relación a los colombianos, (Pedraza, et al., 2015), muestran un patrón de liderazgo similar con tan sólo algunas diferencias en la frecuencia de dichas características y rasgos (Zárate y Matviuk, 2012; Robles, et al., 2013).

A nivel europeo, la evidencia española muestra por ejemplo que a partir del modelo de Meng y Berger (2011) citado por Moreno, et al., (2014), el liderazgo en el sector de las relaciones públicas y la gestión de la comunicación se caracteriza por líderes excelentes, con creencias y percepciones sobre el liderazgo y donde los desafíos que afrontan en la actualidad, así como las estrategias y acciones que implementan, se orientan con pragmatismo a superar con éxito los retos que se presentan en el desempeño de su trabajo.

Extendiendo el análisis hacia las perspectivas de género, los estilos de liderazgo identificados en un grupo de supervisores puertorriqueños, muestra diferencias significativas entre mujeres y hombres. En general, las mujeres presentan niveles superiores de liderazgo transformacional y transaccional, sin embargo, no se encontraron diferencias significativas respecto de las demás variables demográficas (Pérez y Camps, 2011). Las diferencias de género resultan relevantes en tanto se replantean las formas de liderazgo para lograr mayor comunicación, más descentralización, flexibilización en las relaciones con los subordinados y mejor calidad de atención, como se ha demostrado en el ámbito de la gestión de salud (Fardella, 2013), para lo cual resultan esenciales las habilidades diferenciales del liderazgo por género, confirmándose que en ninguna de las habilidades de liderazgo atribuibles al sexo, se pudo demostrar una diferencia estadísticamente significativa entre hombres y mujeres, sin embargo, los atributos flexibilidad, organización y planificación del trabajo, orientación hacia las personas, capacidad de comunicación y de negociación y el trabajo en equipo, mostraron las mayores frecuencias entre las mujeres, en tanto que los hombres atribuyeron más fuertemente la innovación y la visión estratégica (Jorna, et al., 2016).

Por último, desde una mirada más contemporánea y a partir del hecho que las empresas se desarrollan en un contexto de incertidumbre, riesgo, complejidad y dinamismo, que actualmente define una sociedad elusiva, los 
modelos tradicionales de liderazgo parecen no ser suficientes para responder a las necesidades de las organizaciones. Se hace necesario entonces un liderazgo mixto que valora al ejecutor y la ejecución en una complementariedad de sujetos que ejercen un liderazgo líquido que, de manera alternada y conjunta, articulan un nuevo espacio social de trans-disciplinariedad organizacional, donde lo líquido se hace parte de las organizaciones para hacer frente con eficacia a la dinámica de un entorno elusivo (Pereira, 2014; Barbosa, et al., 2014). En este contexto, el objetivo del presente trabajo es determinar las percepciones que los seguidores tienen del liderazgo en el sector de exploración y explotación de minerales no renovables como el oro y otros minerales, en una compañía minera de la Provincia de El Oro al suroeste del Ecuador.

\section{METODOLOGÍA}

Se realizó un diseño cualitativo-cuantitativo con un estudio descriptivo de corte transversal a una población total de 50 colaboradores (Hernández, et al., 2006). El instrumento fue un cuestionario desarrollado por Torres y Contreras (2012), que consta de 45 reactivos en escala Likert (Robbins, 1996; Hernández, et al., 2006) que abordan las dimensiones rasgos de personalidad, percepciones de los subordinados y factores de la cultura que definen el liderazgo (Ruiz, 1998). Para el trabajo de campo se consideró un plan de muestreo probabilístico bietápico, estratificado y por conglomerados, a través de entrevistas personales realizadas desde mayo de 2016, con un total de 50 trabajadores y jefaturas entrevistados.

Se determinó una media de estabilidad laboral de cuatro años y personas con una media de 33 años de edad (Eyssautier, 2006). Dada la naturaleza, procesos, tecnologías y cultura de la industria, un $90 \%$ del personal corresponde al género masculino y un $10 \%$ al género femenino. Se realizó un análisis descriptivo de la muestra mediante el sistema SPSS, seguido de un estudio de correlaciones. Para cada una de las variables de la escala se aplicaron comparaciones de promedios de los sujetos respecto al punto central de la escala (2,5 puntos) utilizando el estadístico $t$ de student para una muestra y, para las diferencias entre grupos, se realizó la prueba de $t$ para muestras independientes. La confiabilidad se verificó mediante el Alfa de Cronbach que mide la consistencia interna de los ítems analizados (Eyssautier, 2006).

Mediante sesiones de grupo foco se identificaron situaciones y contextos laborales asociados a dimensiones y variables relacionadas con el liderazgo recogiéndose percepciones en los términos y palabras expresados por los actuantes para ratificar los reactivos del cuestionario y evitar interpretaciones erradas (Hernández, et al., 2006). Las expresiones finalmente validaron los reactivos y fueron confirmados por los propios participantes. Se verificó la validez de contenido del instrumentos y de los reactivos, sobre la base de diversas teorías (Zárate y Matviuk, 2012; Theime y Treviño, 2012; Robles, et al., 2013; MorelosGomez y Fontalvó-Herrera, 2014; Pedraza, et al., 2015; Contreras, et al., 2016) la de constructo a través de grupos focales, validación con expertos y definición operativa de las variables (Hernández, et al., 2006); la validez convergente, se logró mediante el análisis de factores de componentes principales, con previa verificación de pertinencia del índice KMO y prueba de esfericidad de Bartlett (Eyssautier, 2006), lográndose un índice KMO y esfericidad $(p)$ significativos. Finalmente, la validez discriminante se confirmó por las varianzas explicadas de los factores y las cargas de los componentes con cada factor, superiores o iguales a 0,5 (Eyssautier, 2006).

El pre-testeo del cuestionario para chequear comprensión, lenguaje, formato, instrucciones y escalas de respuesta, y descartar dudas respecto de las preguntas, se realizó con una muestra aleatoria de 25 sujetos (Ruiz, 1998) que incluyó los niveles directivo y empleados de planta de la segunda línea de mando, lo que definió el criterio de exclusión, de modo que no fuesen incorporados en la muestra del estudio general. La validación por expertos se realizó con 4 directivos y 5 académicos, para ratificar lenguaje, unidireccionalidad, estructura semántica, consistencia y validez de las taxonomías teóricas utilizadas (Zárate y Matviuk, 2012; Robles, et al., 2013; Pedraza, et al., 2015; Contreras, et al., 2016). Finalmente, se aplicó el método de reducción de dimensiones a través de un análisis de factores de componentes principales (Eyssautier, 2006) con rotación varimax, verificación de la medida de adecuación muestral de Kaiser-MeyerOlkin (KMO), índice significancia de los datos, verificación de la confiabilidad del instrumento y de las escalas del mismo (Hernández, et al., 2006). El análisis de los datos se realizó por medio del Software IBM SPSS Statistics Ed22 (IBM, 2013).

\section{RESULTADOS}

En esta primera fase y para la totalidad de los reactivos del cuestionario se presentan los resultados del análisis de datos conforme los objetivos del estudio.

\section{Análisis de fiabilidad global}

El primer paso del estudio fue verificar la confiabilidad del instrumento utilizado a cuyo respecto se obtienen los resultados de la Tabla 1. 
Tabla 1: Estadísticos de Confiabilidad y descriptivos para todas las variables

\begin{tabular}{ll}
\hline Estadísticos de fiabilidad & \\
\hline Alfa de Cronbach & 0,891 \\
Alfa de Cronbach basada en los elementos tipificados & 0,899 \\
$\mathrm{~N}$ de elementos & 45 \\
\hline Estadísticos de la escala & \\
\hline Media & 166,36 \\
Varianza & 309,256 \\
Desviación típica & 17,586 \\
\hline
\end{tabular}

El coeficiente alfa de Cronbach al 1\% alcanza 0,899 para la totalidad de los elementos tipificados (45) lo que representa un muy buen indicador de consistencia interna de los datos. Además, para realizar un análisis más detallado de las dimensiones del instrumento, los reactivos se clasificaron en tres grupos, que se muestran en la Tabla 2.

Tabla 2: Confiabilidad de las dimensiones del cuestionario

\begin{tabular}{lccc}
\hline Estadísticas de fiabilidad & $\begin{array}{c}\text { Percepción de los } \\
\text { colaboradores }\end{array}$ & $\begin{array}{c}\text { Rasgos de Personalidad } \\
\text { de los líderes }\end{array}$ & $\begin{array}{c}\text { Cultura } \\
\text { organizacional }\end{array}$ \\
\hline Alfa de Cronbach & 0,793 & 0,692 & 0,769 \\
Alfa de Cronbach basada en los & 0,817 & 0,751 & 0,729 \\
elementos tipificados & & & \\
\hline Estadísticas de escala & 72,44 & 61,34 & 32,58 \\
\hline Media & 70,129 & 33,617 & 34,371 \\
Varianza & 8,374 & 5,798 & 5,863 \\
Desviación estándar & 19 & 15 & 11 \\
N elementos & & &
\end{tabular}

Con una significación al 1\%, los factores de percepción de los colaboradores muestran una confiabilidad de 0,817 para los 19 reactivos que lo conforman. Para los rasgos de personalidad de los líderes con 15 elementos, fue de 0,751 y para los factores de la cultura organización con 11 reactivos fue de 0,729 . Como se observa, en todos los casos los coeficientes representan un índice adecuado de convergencia.

\section{Análisis de factores de componentes principales}

Esta etapa del estudio se ocupó de reducir los reactivos a dimensiones significativas y focalizar el análisis a los elementos más relevantes para dimensionar los componentes que definen el liderazgo como se muestra en la Tabla 3. La medida de adecuación muestral de Kaiser-Meyer-Olkin (KMO) alcanzó índices entre los valores, 0,695, 0,784 y 0,698 para los análisis de los colaboradores, líderes y cultura organizacional. El $\mathrm{KMO}$ para los Factores Excluidos fue igual o inferior a 0,322.

Del análisis se obtuvo cinco factores representativos que incluyen 25 reactivos de los 45 analizados, debiéndose excluir 20 reactivos por no alcanzar los valores adecuados de confiabilidad y de varianza explicada, definidos como mínimos para ser incorporados al estudio. Los cinco factores determinados muestran cargas factoriales de sus componentes altas y varianzas explicadas decrecientes que acumulan un $57,07 \%$ de la varianza total por explicar, razón por la cual el análisis de factores es pertinente, muestra convergencia de datos y focaliza adecuadamente el análisis.

En el primer grupo de factores, el Factor 1, Confianza en el liderazgo, alcanza un 17,08\% de explicación, seguido del Factor 2, Comunicación y apoyo del líder logra un 16,7\% de varianza explicada. Los dos primeros factores explican, por sí solos, un $33,78 \%$ de la varianza total. El segundo grupo de factores, conformado por 3 factores, explican a su vez, un 23,29\% de la varianza en forma adicional. Se observa que, el tercer Factor, Participación y ambiente de trabajo muestra una varianza explicada de 9,35\%, el Factor 4 , Temperamento y personalidad del líder alcanza un $8,86 \%$ y, finalmente el Factor 5, Compañerismo y cercanía, alcanza un 5,08 \% de varianza explicada. Los componentes excluidos, con un $\mathrm{KMO}$, igual o inferior a 0,322, si bien son marginados del presente análisis general de los datos, sí pueden ser incluidos en otros estudios de mayor focalización, por ejemplo, para analizar cada una las tres dimensiones separadamente. Los componentes excluidos se detallan en la Tabla 4. 
Tabla 3: Análisis General de Factores de Componentes Principales. Matriz de componentes rotados

\begin{tabular}{|c|c|}
\hline Factor 1:Confianza en el liderazgo & 17,084 \\
\hline $\begin{array}{l}\text { [FPS30] Mi jefe confía en mí } \\
\text { [FRP31] Mi jefe me estimula para que haga las cosas de manera diferente } \\
\text { [FPS32] Confío en mi jefe } \\
\text { [FRP33] Mi jefe me permite ejecutar ideas innovadoras en mi trabajo } \\
\text { [FRP34] Antes de tomar una decisión, por pequeña que sea debo consultar a mi jefe } \\
\text { [FCO35] Los valores corporativos y/o las competencias, menciona sobre liderazgo de los empleados }\end{array}$ & $\begin{array}{l}0,859 \\
0,764 \\
0,840 \\
0,827 \\
0,749 \\
0,705\end{array}$ \\
\hline Factor 2:Comunicación y apoyo del líder & 16,274 \\
\hline $\begin{array}{l}\text { [FPS03] [Mi jefe da confianza } \\
\text { [FPS11] Mi jefe sabe escuchar } \\
\text { [FPS14] Mi jefe es un ejemplo a seguir } \\
\text { [FPS15] Mi jefe me apoya en el trabajo } \\
\text { [FPS17] A mi jefe le gusta enseñarme para que yo sea una mejor persona } \\
\end{array}$ & $\begin{array}{l}0,615 \\
0,696 \\
0,684 \\
0,632 \\
0,624 \\
0,685\end{array}$ \\
\hline Factor 3:Participación y ambiente de trabajo Varianza Explicada & 9,350 \\
\hline $\begin{array}{l}\text { [FCO38] A través de esta empresa he participado en actividades para desarrollar competencias de } \\
\text { liderazgo } \\
\text { [FCO39] El ambiente de trabajo en esta empresa es agradable } \\
\text { [FCO40] La empresa nos da oportunidad de ascenso } \\
\text { [FCO41] En esta empresa se pueden generar cambios rápidos }\end{array}$ & $\begin{array}{l}0,645 \\
0,572 \\
0,670\end{array}$ \\
\hline Factor 4:Temperamento y personalidad del líder & 8,863 \\
\hline $\begin{array}{l}\text { [FRP08] Mi jefe es temperamental, de humor cambiante } \\
\text { [FPS23] Le tengo miedo a mi jefe } \\
\text { [FRP26] Mi jefe me ofende cuando me equivoco } \\
\text { [FPS28] Mi jefe me da reconocimiento (felicita o incrementa sueldo) } \\
\text { [FCO43] Mi jefe está enfocado en mis errores y defectos } \\
\end{array}$ & $\begin{array}{l}0,503 \\
0,621 \\
0,701 \\
0,596 \\
0,563 \\
0,645\end{array}$ \\
\hline Factor 5:Compañerismo y cercanía & 5,084 \\
\hline \multicolumn{2}{|l|}{ KMO y prueba de Bartlett } \\
\hline $\begin{array}{ll}\text { Medida de adecuación de Kaiser-Meyer-Olkin (KMO). } \\
\begin{array}{ll}\text { Prueba de esfericidad de Bartlett } & \text { Chi-cuadrado aproximado } \\
& \text { Gl } \\
& \text { Sig. }\end{array}\end{array}$ & $\begin{array}{r}0,784 \\
1893,245 \\
990 \\
, 000 \\
\end{array}$ \\
\hline
\end{tabular}

Tabla 4: Componentes excluidos

\begin{tabular}{|c|c|}
\hline [FRP01] Mi jefe es extrovertido y sociable & $\begin{array}{l}\text { [FPS22] Mi jefe nos entusiasma para que hagamos el } \\
\text { trabajo con gusto }\end{array}$ \\
\hline [FRP04] Mi iefe es honesto & [FPS24] Mi jefe me habla sobre lo importante de alcanzar \\
\hline [FRP04] Mi jefe es honesto & una visión en el futuro \\
\hline$[\mathrm{FH}$ & [FPS25] Mi jefe me da instrucciones claras \\
\hline$[F$ & [FPS27] Puedo hablar con mi jefe en cualquier momento \\
\hline [FRP10] mi jefe le facilita hacer cambios & $\begin{array}{l}\text { [FCO36] Un requisito para cargos directivos en esta } \\
\text { empresa, debe tener competencias de liderazgo }\end{array}$ \\
\hline [FRP12] Mi jefe entiende las emociones de los demás & [FCO37] Un requisito para cargos operativos en es \\
\hline [FRP13] M & empre \\
\hline & [FCO42] En esta empresa las normas y reglas se deben \\
\hline [FPS16] Mi jefe me escucha con atención & \\
\hline $\begin{array}{l}\text { [FPS21] Pregunto a mi jefe cuando no sé, para que me } \\
\text { enseñe }\end{array}$ & $\begin{array}{l}\text { [FCO44] Frecuencia para desarrollar mis competencias de } \\
\text { liderazgo es? }\end{array}$ \\
\hline & [FCO45] Programas o actividades que ha participado \\
\hline
\end{tabular}

Interpretación y significados de los factores

El Factor 1, confianza en el liderazgo, que alcanza un 17,08\% de explicación, hace referencia a los rasgos de percepción del líder. El $76 \%$ está de acuerdo y muy de acuerdo que los seguidores confían en sus jefes y recíprocamente en el mismo porcentaje, los jefes confían en los colaboradores. El $70 \%$ de los colaboradores están de acuerdo e indican que el jefe los estimula para que haga las cosas de manera diferente y manifiestan que sus jefes les permiten ejecutar ideas innovadoras en sus trabajos (72\%) y que, antes de 
tomar una decisión, por pequeña que sea, deben consultar a sus jefes (74\%). En este último caso, aunque hay confianza entre líder-colaborador y viceversa; existe una relación de dependencia en algunos tipos de trabajo operativos por seguridad, como es el hecho de ubicar explosivos en lugares adecuados.

Por otra parte, el Factor 2, comunicación y apoyo del líder con un 16,7\% de varianza explicada, se reporta que el $76 \%$ de los subordinados aprecia que el jefe sabe escuchar y en el mismo porcentaje el jefe es un ejemplo a seguir. Mientras que el $72 \%$ está de acuerdo, y muy de acuerdo, que el jefe les apoya en el trabajo. El 70\% indica favorablemente que: A mi jefe le gusta enseñarme para que yo sea una mejor persona. Así mismo, el tercer Factor, participación y ambiente de trabajo, que muestra una varianza explicada de 9,35\%, describe que: A través de esta empresa he participado en actividades para desarrollar competencias personales de liderazgo.

El Factor 4, temperamento y personalidad del líder con un 8,86\% de varianza explicada, se enfoca en que mi jefe me ofende cuando me equivoco, lo que demuestra que debido a las actividades desarrolladas en las bocaminas, no se permite errores, los cuales podrían ser fatales en accidentes de trabajo. Finalmente, el Factor 5, compañerismo y cercanía, con un 5,08\% de varianza explicada, centra el análisis en que mis compañeros y yo somos igual de cercanos a mi jefe lo que da como resultado la percepción que el jefe sería una persona de trato igualitario.

\section{CONCLUSIONES}

De la investigación realizada y del análisis de datos resultante se obtienen las siguientes conclusiones: i) Que los colaboradores tienen confianza en los rasgos de personalidad de sus líderes al tiempo que tanto seguidores como jefes confían recíprocamente entre sí: ii) Perciben la participación que se les otorga y la valoran porque genera un buen ambiente de trabajo y además, porque sus jefes se relacionan con ellos mediante un trato igualitario. Esto se expresa a través de mecanismos de comunicación con sus jefaturas y porque perciben el apoyo de sus líderes cuando les permiten ejecutar ideas innovadoras en sus trabajos; y iii) Perciben la existencia de una relación de dependencia en algunos tipos de trabajo operativos a causa de la seguridad requerida, como es el hecho de ubicar explosivos en lugares adecuados. Esto se expresa mediante la firmeza de carácter, temperamento y personalidad de sus respectivos jefes, señalando que, aunque a veces podrían sentirse ofendidos, lo explican y comprenden por tener que enfrentar actividades de alto riesgo.

\section{REFERENCIAS}

Avolio, B. J. y Bass. B. M., Multifactor leadership questionnaire: Manual and sample set, $3^{\text {rd }}$ Ed. Redwood City, ca: Mind Garden (2004)

Barbosa, R. D. H., Mihi R. A., Noguera H. Á., Knowledge management and leadership: Relationship prospects. Perspect. Psicol. ISSN: $1794-9998$ (en línea), 10 (1) 057-070 (2014). https://goo.gl/2Tgi4V. Acceso: 27 de febrero de (2017)

Bass, B. M., Avolio, B. J., Jung, D. I. y Berson, Y., Prediction unit performance by assessing transformational and transactional leadership. doi.org/10.1037/0021-9010.88.2.207. Journal of Applied Psychology 88(2), 207-218 (2003)

Borjas, De Xena, L., The Leadership Competency In Venezuelan Managers. Rev. Fac. Cienc. Econ., 19 (2) Bogotá, July / Dec. (2011)

Cabaleiro G., Jiménez, A., Miles, J. y Horta R., Liderazgo e innovación para un mundo más sostenible. Journal of Technology Managemente \& Innovatión, ISSN 0718-2724 (en línea): 11 (1) Santiago 2016. https://goo.gl/J12vXN. Acceso: enero de (2017)

Contreras P. O., Vesga M. A. y Barbosa C. A., Frames of Leadership Into the Companies: The Case of The City of Bucaramanga (Colombia) And Its Sourounded Area. Dimensión Empresarial, 14 (1) $39-55$ Enero Junio de (2016)

Duran, M. G. y Castañeda D. I., Relación entre Liderazgo Transformacional y Transaccional con la Conducta de compartir conocimiento en dos empresas de servicios. Acta Colombiana de Psicología, 18.1 (2015)

Dvir, T., Eden, D., Avolio, B. J. y Shamir, B., Impact of transformational leader ship on follower development and performance: A field experiment. doi.org/10.2307/3069307. Academy of Management Journal, 45(4), 735-744 (2002)

Ehrhart, M.G., Leadership and procedural justice climate as antecedents of unit-level organizational citizenship behavior. doi.org/10.1111/j.1744-6570.2004.tb02484.x. Personnel Psychology, 57, 61-94 (2004) 
Escalona-Barbosa, D., Hurtado-Ayala, A., Influencia de los estilos de liderazgo en el desempeño de las empresas exportadoras de colombia. Estudis Generales, 32.139 (2016)

Espinosa, J., Contreras F. y Barbosa, D., Prácticas de Liderazgo y su relación con la Cultura en un grupo de paises Latinoamericano, 11.2 (2015)

Eyssautier, M., Metodología de la investigación. México D.F.: Editorial Thomson (2006)

Fardella R. J., Habilidades estratégicas del líder. Strategic skills of leaders. Revista Cubana de Enfermería, 29(3), 233-243 (2013)

Garcia, S. M., Papel de los seguidores en el desarrollo de las teorías de liderazgo Organizacional. Apuntes del Cenes, 34.59 (2015)

Gil, O. I. e Ibarra, L. S., Impact Of Leadership In Critical Success Factors As A Competitive Business Strategy. Dimensión Empresarial / 12 (2) 117-126, Julio - Diciembre (2014)

Gil, O., I. e Ibarra, L. S., Incidencia del liderazgo en los factores críticos del éxito como estrategia competitiva empresarial. Dimension Empresarial, 12.2 (2014)

Godoy, R., y Bresó, E., ¿Es el liderazgo transformacional determinante en la motivación intrinsica de los seguidores? Revista de psicología del trabajo y de las organizaciones, 29.2 (2013)

Hernández, R., Fernández, C. y Baptista, P., Metodología de la Investigación. México D. F.: Mc Graw-Hill (2006)

IBM. SPSS Statistics Base 20v (2013)

Jorna, C.A., Castañeda, A.I. y Veliz, M.P., Perception of leading skills by men and women for the health management in Cuba. Rev. Cubana Salud Pública, 42 (1) Ciudad de La Habana enero-marzo (2016)

Li, A. N. y Liao, H., How do leader-member exchange quality and differentiation affect performance in teams? An integrated multilevel dual-process model. Journal of Applied Psychology, 99, 847-866 (2014)

Liao, H. y Chuang, A., A multilevel investigation of factors influencing employee service performance and customer outcomes. Academy of Management Journal, 47, 41-58 (2004)

Lim, B. y Ployhart, R., Transformational leadership: Relations to the five-factor model and team performance in typical and maximum contexts. Journal of Applied Psychology, 89, 610-621 (2004)

Luthans, F., Avolio, B., Walumbwa, F. y Li, W., The Psychological Capital of Chinese Workers: Exploring the Relationship with Performance. Management and Organization Review, 1, 249-258 (2005)

Mendoza de Ferrer, H., Modernism and Postmodernism, Toward the Construction of Anthropocentric Leadership. Revista Venezolana de Gerencia, 12 (44) Maracaibo dic. (2008)

Morelos-Gomez, J. y Fontalvó-Herrera, T., Análisis de los factores determinantes de la Cultura Organizacional en el Ambiente Empresarial, 10.1 (2014)

Moreno, M., Navarro, C. y Humanes, M., El liderazgo en relaciones públicas y gestión de comunicación. Análisis cuantitativo de los factores de liderazgo en el sector en España. Palabra Clave, 17 (3) Chia Sept. / Dec. (2014)

Ochoa, D. H. y Ríos, M. A., Liderazgo, la competencia esencial que transformó una empresa colombiana en un gigante internacional: el caso de Cementos Argos. Estudios gerenciales, 27(121) 39-58 Octubre Diciembre (2011)

Pautt T. G., Liderazgo y Dirección: Dos Conceptos Distintos con Resultados Diferentes. Rev. Fac. Cienc. Econ., ISSN: 0121-6805 (en línea): XIX (1), Junio, 213-228 (2011). https://goo.gl/2GOXMT. Acceso: diciembre de (2016)

Pedraza, M. N., Lavín V. J., Delgado R. G. y Bernal G. I., Prácticas de Liderazgo en Empresas Comerciales En Tamaulipas (México). Revista Facultad de Ciencias Económicas, XXIII (1), 251-265, Junio (2015)

Pedroja-Rejas, L. y Rodriguez-Ponce E., Estudio comparativo de la influencia del estilo de liderazgo y la congruencia de valores en la eficacia de empresas privadas e instituciones públicas. Interciencia, 33.1 (2008)

Pereira S. A., Liquid leadership: a proposal to face uncertainly and risk. Pensam. gest. no.37 Barranquilla July/Dec. (2014)

Perez L. S., La influencia del liderzgo sobre el aprendizaje. El papel mediador del contexto organizativo. Innovar, 22.45 (2012) 
Perez-Rave, J., La Rotta, D. y Lopez, K., Predictores de Excelencia desde la Voz del Empleado (EVE) en un Centro Médico. Doi: 10.4067/S0718-07642013000500006. Inf. Tecnol., 24 (5), 41-50 (2013)

Pérez, S. J. y Camps V., Transformational Leadership Manifestation in a Group of Puertorrican Supervisors. Rev. Psicol. trab. organ., 27 (1) Madrid abr. (2011)

Perez, J. y Camps, V., Manifestación del liderazgo tranformacional en un grupo de supervisores de Puerto Rico. Revista de Psicología del Trabajo y de las Organizaciones, 1(27) 17-29 (2011)

Peterson, S. J. y Luthans, F., The positive impact and development of hopeful leaders. Leadership and Organization Development Journal, 24, 26-31 (2003)

Piccolo, R. F. y Colquitt, J. A., Transformational leadership and job behaviors: The mediating role of job characteristics. Academy of Management Journal, 49, 327-340 (2006)

Robbins, Stephen P., Comportamiento Organizacional. Teoría y Práctica. Prentice Hall, $7^{a}$ Ed. (1996)

Robles F. V., Contreras T. F., Barbosa R. D. y Juárez A. F., Leadership in Colombian mexican managers: A comparative study. Investig. Desarro. 21 (2) Barranquilla July / Dec. (2013)

Rodríguez, E., Estilos de liderazgo, cultura organizativa y eficacia: un estudio empírico en pequeñas y medianas empresas. Revista de Ciencias Sociales, 16 (4) Maracaibo dic. (2010)

Ruiz, C., Instrumentos de Investigación Educativa. Barquisimeto, CIDEG (1998)

Salinas M. P. y Cordero Ch. C., Transformación de los liderazgos en la Mineria: gestión estrategica para incorporar mujeres a la industria. Innovar, 26.61 (2016)

Seibert, S. E., Wang, G. y Courtright, S.H., Antecedents and consequences of psy-chological and team empowerment in organizations: A meta-analytic review. doi.org/10.1037/a0022676. Journal of Applied Psychology, 96, 986-1003 (2011)

Terroni, N., El liderazgo en grupos y organizaciones. Una revisión teórica. Revista Idea, 16 (37), 75 - 84 (2002)

Theime, C. y Treviño, E., Liderazgo en Educación: Al final sólo el carisma importa. Espacio Abierto 21(1) 3755 (2012)

Torres, G. y Contreras, C., Factors that influence leadership of the financial sector managers in the city of Cucuta, Norte de Santander. Rev. esc.adm.neg. ISSN 0120-8160, (en línea), no.73 Bogotá July/Dec. 2012 https://goo.gl/1T1sMy. Acceso: 5 de marzo de (2016)

Turbay-Posada, M., Liderazgo e Innovación Organizacional. Psicología desde el caribe 30.1 (2013)

Walumbwa, F., Wang, P., Lawler, J. y Shi, K., The role of collective efficacy in the relations between transformational leadership and work outcomes. doi.org/10.1348/0963179042596441. Journal of Occupational and Organizational Psychology, 77, 515-530 (2004)

Wang, M., Liu, S., Liao, H., Gong, Y., Kammeyer-Mueller, J. y Shi, J., Can't get it out of my mind: Within- and between-person relationships between employee rumination after customer mistreatment and negative mood. Journal of Applied Psychology, 98, 989-1004 (2013)

Wegner, L., Organizational leaders and empowered employees: The relationship between leadership styles, perception of styles, and the impact on organizational outcomes. Capella University, From ProQuest database (2004)

Whetstone, J.T., Personalism and moral leadership: the servant leader with a transforming vision. doi:10.1111/1467-8608.00298. Business Ethics: A European Review, 11, 385-392 (2002)

Zárate T. R. y Matviuk S., Emotional intelligence and leadership practices in Colombian Organizations. Cuadernos de Administración, Universidad del Valle, 28(47) enero - junio (2012) 